course in cases which when seen are practically hopeless, or at any rate too advanced apparently to do any good to by attempting an operation.

2. That of trying to reduce the gut by means of the distension of the bowel below the invagination, either with liquid or gas, and helped in some cases by manipulation through the abdominal walls. I shall discuss this under three practical headings :- (a) Cases suitable for this line of treatment. (i.) They must be recent. It will be but rarely successful, I am disposed to believe, after forty-eight, or at the most fifty-four hours from the commensement of the acute symptoms. (ii.) It is not satisfactory to employ it unless a definite tumour has been observed, otherwise it would be extremely difficult to he sure of the effect of the injection, at any rate immediately. (iii.) It would hardly be likely to be of any benefit if the invaginated bowel were already in the rectum. (b) The choice between gas and liquid. I am strongly in favour of the latter, for the following reasons: (i.) Liquid is much more easily introduced into, and retained within the bowel. (ii.) It can be injected with great exactness as regards force and quantity. (iii.) Its greater density will probably have some action in more effectively reducing the gut. (iv.) That the statistics show a decidedly better result with liquid than with gas. If gas be chosen, what gas is used is quite a minor detail. Lately a successful case of reduction has been recorded, in which carbonic acid gas was generated within the bowel by the action of acid upon a carbonate. If liquid be decided upon it may be water, oil, milk, or gruel, of which I think milk is as good as any, if it be at hand. (c) The method of injection : (i.) The liquid should be of the temperature of 99 deg. F. while being injected. (ii.) The child should always, if possible, be anæsthetised with chloroform. This, I believe, is a very important detail, for not only does it efface pain, but allows a much greater quantity of fluid to be injected, and what is of prime importance, allows the surgeon to easily notice and feel the effect of the injection upon the tumour. (iii.) The liquid may either be introduced with a Higginson's syringe, or by hydrostatic pressure. The latter is the better way, the reservoir not being raised higher than three feet. (iv.) The child may be partially or wholly inverted during the process, and manipulation may be applied through the abdominal walls. ( $\nabla$.) The patient must be protected against exposure. (vi.) Small doses of opium should be administered subsequently to reduction.

A word of warning must here be given. In many cases the tumour is apt to become impalpable, owing to its having disappeared up under the liver or elsewhere, and this must not be mistaken for its complete reduction. Often the condition of invagination is said to recur after reduction by fluid. I believe most of such cases are really instances of incomplete restora- tion of the bowel to its normal condition, for the last portion of the intussusceptum is often very difficult to reduce, and may be the starting point for a relapse into a state quite as bad as before.

(3). Lastly, we have the treatment by laparotomy and so reducing the invagination by direct manipulation; or, if this be impossible, of otherwise dealing with the gut. If one careful and thorough attempt at reduction by injection fail to completely push back the gut, a fact which is often uncertain but evidenced either by the continuation of the symptoms or better the presence of the tumour, I feel sure the best plan is, without further delay, to open the abdomen as a rule. In performing laparotomy under these circumstances the following points should be borne in mind: (i.) To operate promptly, but not hurriedly. (ii.) To very carefully protect the young patient from all unnecessary exposure during the operation by keeping the limbs wrapped in cotton wool and having the infant laid upon a hot-water cushion. (iii.) The incision is probably best commenced in the middle line above the umbilicus, but may have to be enlarged downwards. (iv.) As soon as the abdomen has been opened the fingers of the operator should be inserted and the tumour felt for, and brought to the surface if possible. (v.) Reduction should then be effected by squeezing the gut out rather than by dragging, though the latter may be gently employed. In a large number of cases, especially if early, the gut is easily reduced, except perhaps the very last portion, chiefly in the ileo-cæcal variety. This is due to the highly œdematous condition of the part. Firm pressure around the circumference of the gut with the hand might aid in freeing this portion. (vi.) When reduction has been brought about, any escaped small intestine will have to be returned, and this is most easily accomplished by the edges of the abdominal wound being held up, and so to speak being broughtover the gut, rather than the bowel being forced back into the abdomen. Great care must be taken that any exposed intestine is not chilled. (vii.) The wound is now rapidly closed, and the child returned to a thoroughly warmed bed in front of a fire, and minute doses of opium given every few hours. (viii.) If reduction cannot be effected, probably the best line of treatment is to establsh an artificial anus by stitching and opening the bowel above the invagination. Such cases, however, usually end fatally.

The results of laparotomy even in very young subjects have of late years been highly encouraging, but it still remains for every practitioner to diagnose his cases early, and then to treat them as above indicated promptly if he would have good success.

I am sure the day is not far distant when the mortality of over 70 per cent. of acute cases will be reversed, with a percentage of recoveries exceeding our most sanguine anticipations.

\title{
Medical Progress.
}

FEVERS AND INFECTIOUS DISEASES.

On the Production of Fever.-Hale White, ${ }^{1}$ writing on the theory that all pyrexia is essentially neurotic, considers that a rise of temperature may be due either to direct interference with the thermic centres or to the circulation through them of toxic blood or to reflex stimulation of them. As instances of the last case, he gives the pyrexia seen in gallstone colic, in simple fractures, and where pus under tense fasciæ causes effects far out of proportion to its quantity. Specific toxins, or full doses of caffeine or belladonna, causes pyrexia through the blood. As to direct action, experimental injury to the corpus striatum in rabbits causes pyrexia, while injury to the cortex an erratic and irregular form. $\mathrm{He}$ considers the thermogenetic centres are situated in the former, and the thermotaxic: in the latter. Clinical records bear out this view, and he notices that if one corpus striatum be injured the 
temperature is usually higher on the opposite side of the body, and that the pyrexia from a striate lesion attains its maximum in twenty-four hours, and is followed by a subnormal fall. In brain hæmorrhage there may be a primary fall from shock. Perversion of the thermotaxic mechanism is shown in laceration of the cortex, meningeal hæmorrbage, and meningitis. In the latter the temperature is erratic, and may reach a high degree, but it bears no relation to the pulse and respiration. Hence, probably, the amount of heat produced by the body is not correctly expressed by the rise of temperature. In epilepsy, chorea, and hysteria there is functional interference with the cortex, and in each disturbance of the tem. perature may occur. This is marked in the status epilepticus. A rise is found at times immediately after epileptic fits, and when occurring in hysteria the temperatures are erratic, are often not related to the pulse and respiration, are affected very little by antipyretics, and may vary in different parts of the body. Injuries of the cord and pons produce pyrexia by interference with the transmitting fibres, and finally those pyrexiæ produced through the thermolytic centres (vasomotor and sweat) are well recognised.

C. C. Easterbrook ${ }^{2}$ defines pyrexia as a morbid elevation of temperature, and fever as a derangement of the quantity of heat in the body. Either can exist without the other. Heat production depends on the nerve control of metabolism. The theor $y^{3}$ that fever depends essentially on an increase of heat production has been advanced by Hiller, while Kraus, of Vienna, claims to have proved by calorimetry that a rise of temperature is accompanied by diminished radiation, and that the vessels of a fever patient are alternately contracted and dilated. Occasionally fevers, such as scarlet ${ }^{4}$ or typhoid, run their course with subnormal temperatures. Teissier ${ }^{5}$ relates a case of pneumonia of this kind, and thinks that such instances are due to the retention of the products of cellular nutrition, under the influence of the specific poison. An analagous case is presented by the subnormal temperatures sometimes found in gout. Dr. Teissier employs quinine in these interesting cases, and remarks that it raises the temperature. D'Arsonval ${ }^{6}$ and Charrin ${ }^{7}$ lay down that (1) microbes act by their products both on the central heat and radiation, increasing the one and diminishing the other. (2) Pyrexia maybe caused by substances producing vaso constriction, as well as by those producing vaso dilatation, pyocyanic toxins, and tuberculin. Hence the inadequacy of a vasomotor theory of fever. (3) Calorimetric observations can alone show the pathology of fevers. Over production of heat may exist while the thermometer shows either a great fall or rise of temperature. (4) They have found substances which diminish production of heat and yet raise peripheral temperatures, and others actually increase the temperature while they increase radiation.

The ordinary hectic fever is universally associated with the presence of pus generating organisms, and is characterised by a cold, a hot, and a sweating stage. The regularity of these stages is important in the diagnosis of hectic, but the quantity of the perspiration bears a very variable relation to the severity of the disease or to the height of the temperature. The difficulties of diagnosis with regard to malarial diseases is discussed in a paper by H. S. Stark, of New York $^{8}$. As to pathology, he considers it to be the result of the poisoning of nerve centres by the products of strepti and staphylococci. This nerve element is shown in the flush and sweats, which are such marked features of the disease, and are typical vaso-motor effects also.

An important paper on nerve debility in the production of fever was read at the International Congress by Professor Bouchard, ${ }^{9}$ who calls attention to the rise of temperature caused in debilitated persons by sudden emotion, by the first meal of solid food in an illness, the visits of friends, or removal to a hospital; and holds that these are simply instances where, owing to the instability of the nervous system, the heat producing or destroying functions are easily set in motion. From the experiments of Liebermeister and himself he believes that even moderate muscular effort would produce a great rise of temperature were it not controlled by the thermotaxic mechanism, which is both prophylactic and curative. Some rise even in health is produced by any exercise of the muscles, but this is quickly checked by automatic action. Thus external cold produces shivering and muscular tremor, with destruction of cell contents, tending to increase the production of heat, and, on the other hand, it contracts the superficial vessels and stops evaporation from the sweat glands. While reflexes thus defend us from external cold and heat, the internal heat is regulated directly by the nervous centres, as their own temperature is affected by the temperature of the deeper parts of the body. Whenever, then, the nerrous system is weakened the ordinary forces which produce or dissipate heat are seen as under a magnifying glass. Thus we may have pyrexia from muscular work, emotion, or even from intellectual effort, when the ordinary restraining forces are in abeyance.

In a paper on hyperpyrexia Dr. Willoughby ${ }^{10}$ argues that abnormally high temperatures must be controlled to prevent rapid granular degeneration of the heart and other tissues, either by drugs which decrease oxidation and favour heat elimination, or by the application of cold. The latter is by far the most successful, not only regulating heat, but soothing the injured nervous system.

The number of fevers which are found to be of bacterial origin is constantly increasing, and great interest has been created by the proof of the influence of light in destroying microbic life. Professor Marshall Ward ${ }^{11}$ shows that the blue rays of the spectrum shall Ward shows thers but Khmelevsky found that retardation was produced even by electrical light, and that the effect of solar rays by electrical light, and that the expect to them. It is disputed whether thermal rays have this power, and the possibility of utilising sunlight as a disinfecand the possibility of utilising which are damaged by moist heat, has been disproved by the elaborate experiments of Von Esmarch. ${ }^{12}$

(1894, 3 Am. J. Med.

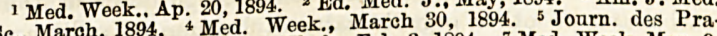
ticiens, Feb, 7, 1894. 6 Med. Week., Feb. 3, 1894. 7 Med. Week, Mar. 9 ticis 8 Med. Rec.. Ap. 14, 1894. 9'Med. Week., Ap. 6, 1894 . 10 Lancet March 10, 1894. ii B. M. J., May 5, 1894. ${ }^{12}$ Zeit. J. Hyg. xvi., B 2. 